\title{
RF Channel Modeling for Implant to Implant Communication and Implant to Sub- Cutaneous Implant Communication for Future Leadless Cardiac Pacemakers
}

\author{
Pritam Bose, Ali Khaleghi, Senior Member, IEEE, Mohammad Albatat, Jacob Bergsland, Ilangko \\ Balasingham, Senior Member, IEEE
}

\begin{abstract}
Propagation of radio-frequency (RF) signals inside human body is demanding to analyze as it is a highly complex medium consisting of different frequency-dependent lossy materials of varying thickness. Moreover, experimental analyses are also unfeasible because that requires probes to be placed inside a human body to collect the signals. This paper focuses on in-body to in-body implant communication for future multi-nodal capsulelike leadless cardiac pacemaker technology. The frequency range of 0.3 - $3 \mathrm{GHz}$ is analyzed using very detailed numerical simulations of digital human models. The results show that the Industrial, Scientific, and Medical (ISM) radio band of the frequency range of $2.4-2.5 \mathrm{GHz}$ is optimal, having the least attenuation of signals considering the size constraints of the implant antenna. Furthermore, the placement of an additional sub-cutaneous implant transceiver is studied. The analysis shows that the abdominal wall is the optimal position for the placement of the implant compared to shoulder and lateral side of the body. This result is further validated by an in-vivo experiment on an adult pig. The other novelty of the study is the investigation of the channel behavior based on ventricular blood volume of the heart to find out the appropriate timing of the transmission of signals between the implants. The results show that the attenuation of the signal increases with the increase in blood volume inside the heart.
\end{abstract}

Index Terms - Implants, pacemaker antenna, wireless communication

\section{INTRODUCTION}

$\mathrm{T}$ HE functional sophistication and complexity of implantable medical device systems have increased over the years. It has become increasingly more important for such systems to include a system for facilitating communication between one

This work was funded by the European Union's H2020: MSCA: ITN program for the "Wireless In-body Environment Communication - WiBEC" project under the grant agreement no. 675353 .

Pritam Bose and Mohammad Albatat are with the Intervention Center, Oslo University Hospital, NO-0027 Oslo and also with the Faculty of Medicine, University of Oslo, 0315, Oslo, Norway (e-mail: pritam.bose@ studmed.uio.no and mohammad.albatat@studmed.uio.no)

Jacob Bergsland is with the the Intervention Center, Oslo University Hospital, NO-0027 Oslo, Norway, and BH Heart Center, Tuzla, Bosnia and Herzegovina (email: jacobbergsland622@gmail.com).

Ali Khaleghi and Ilangko Balasingham are with the Intervention Center, Oslo University Hospital, NO-0027 Oslo and with the Department of Electronic Systems, Norweigian University of Science and Technology, 7491 Trondheim, Norway (e-mail: ali.khaleghi@ @r-

research.no and ilangko.balasingham@medisin.uio.no). implanted device and another and/or an external device, such as a programming console, monitoring system, or the like [1]. Knowledge of the wave propagation media is a key step towards a successful design of a wireless communication system. Such information is typically gathered by conducting physical experiments, measuring and processing the corresponding data to obtain channel characteristics. In case of medical implants, understanding this propagation medium is rather difficult $[2,3]$. Thus, software simulation is one of the most substantial techniques to have an estimated knowledge of the channel characteristics. To the best our knowledge, very little research has been done on characterizing the channel models for in-body to in-body communication compared to conventional communications outside the body [4-6]. Since human body consists of many different types of tissues with varying electromagnetic properties, the channel model varies correspondingly depending on the positions of the implants inside the body.

The Federal Communication Commission (FCC) has allocated the frequencies in the $402-405 \mathrm{MHz}$ range to be used for Medical Implant Communication Services (MICS). This is an ultra-low power, unlicensed mobile radio service for transmitting data in support of diagnostic or therapeutic functions associated with implanted medical devices [7]. The MICS permits individuals and medical practitioners to utilize ultra-low power medical implant devices, such as cardiac pacemakers and defibrillators, without interfering with other users of the electromagnetic radio spectrum. Among primary reasons for selecting these frequencies, one can point to better propagation characteristics for medical implants, reasonable sized antennas, and worldwide availability [8,9]. However, the MICS frequency band may not always be the appropriate option depending on the implant position and the required antenna size. This paper mainly focuses on in-body RF channel modeling to investigate and develop communication framework for pacing/ resynchronization of the heart using multiple spatial heart sensor nodes. The commercially available leadless pacemaker technology offers single-chamber stimulation but the technology providing multi-chamber stimulations and cardiac resynchronization will be an optimum solution. When building a practical communication model, the 


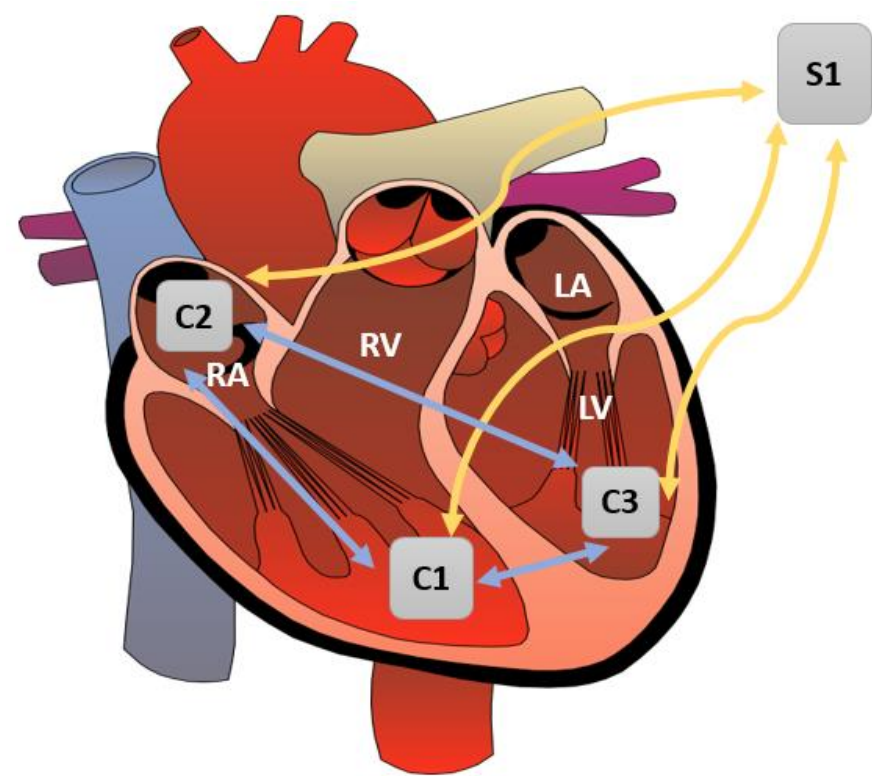

Fig. 1 Schematic of a three-node leadless capsule pacemaker application for cardiac resynchronization therapy. The capsules $\mathrm{C} 1, \mathrm{C} 2$ and $\mathrm{C} 3$ are placed inside the chambers of the heart and they can communicate with the subcutaneous implant S1. RA- right atrium, RV- right ventricle, LA- left atrium, LV- left ventricle, C- capsules and S- subcutaneous implant

optimal frequency band for our multi-node leadless cardiac multi-node leadless cardiac pacemaker application (see Fig. 1) is evaluated based on the size of the capsule pacemakers and the corresponding channel responses.

The study involves modeling of in-body communication channels within the human heart as well as from the heart to different areas surrounding the heart using mathematical simulations. One of the aims is to determine the appropriate frequency for operation of our conceptual cardiac capsule pacemakers optimizing the power consumption of the signal transmission among the capsules. Considering an appropriate capsule size, to minimize the chance of interference with mechanics and hemodynamics of the heart, our experiments have shown promising results at the ISM radio band. This finding is based on using a simple dipole antenna with length of $5 \mathrm{~mm}$ and a diameter of $2 \mathrm{~mm}$. The length of $5 \mathrm{~mm}$ is a rational size for the antenna, whereas the rest of the space of the capsule will be occupied by other components. For the capsule to be implanted transvenously using current catheters, the length of the capsule is limited to $10 \mathrm{~mm}$ for the multi-node pacemaker technology. The size of required antennas and battery restricts available space for sensors and actuators to be incorporated in a such device, which often necessitating a trade-off between bandwidth for transmission, data rates, power requirements and the size of the device. This paper also focuses in finding the ideal position for a sub-cutaneous implant based on simulation results and animal experiments.

The other novelty of the paper is the analysis of the channel behavior with varying ventricular volume i.e. ventricular blood volume variations during the cardiac cycle $[10,11]$. During end-diastole, the heart has the maximum amount of blood as it is filled whereas, during end-systole, the heart has the lowest amount of blood as most of the blood is ejected. Various scenarios with different ventricular blood volume are analyzed to determine the appropriate timing of the transmission of signals between the implants. To best of our knowledge, no significant research has been done on analyzing the variation of received signal-strength with cardiac cycle.

The following sections of the paper are organized as follows: Section 2 describes the methods used in the study and the simulations constructed to study RF propagation from radio implants. Description of our simulation results is provided in Section 3. The in-vivo animal experimental setup and results are described in Section 4. Finally, concluding remarks and future directions are expressed in Section 5.

\section{METHODOLOGY}

The human body is not an ideal medium for transmissions of RF wave. Understanding the effect of human body on the RF signal is essential when designing an in-body data communication link. Unlike standard communications through air, the various tissues and organs within the body have unique conductivities, dielectric constants, and characteristic impedances. Therefore, depending on the frequency of operation, the human body can cause high losses due to power absorption and radiation pattern destruction [12, 13]. The absorption effects vary in magnitude with both frequency of applied field and the characteristics of the tissue, which is largely based on the water and ionic contents. It is very demanding to determine the absorption of the electromagnetic power radiated from an implanted source by the human body [14]. Understanding the human body's effect on RF wave propagation is complicated by the fact that the different components of the body offer varying degrees and types of RF interaction. Although several investigations have been conducted to determine the effect of human body on radiated field [15-19], most of the studies have been based on external sources. The liquid nature of most body structures gives a degree of RF attenuation, whereas, the skeletal structure introduces wave diffraction and refraction at certain frequencies [20]. When the wavelength of a signal is significantly larger than the cross-section of the human body being penetrated, there is very little effect on the signal. However, the human body begin to introduce a scattering effect on the RF signal with increase in frequency [21]. To construct a reliable wireless communication link from/to the human body, the body must be characterized as a medium for wave propagation. And to do so, the electrical properties of the body tissues, mainly the dielectric properties, should be known for the frequency of interest.

The dielectric constant describes how a material is affected by electric fields and is usually described as a dimensionless ratio in terms of the permittivity of free space [22]. The dielectric constant can vary substantially with frequency and can therefore only be considered constant for a finite range of frequencies. Conductivity describes how much the electromagnetic wave is attenuated as it transits the body tissues. Conductivity diminishes electromagnetic radiation as the free electrons and ions in the material are moved by the incoming wave's fields. The electrons and ions will seek the lowest energy state and rearrange themselves in such a way that cancel out the incoming wave's field. If the electrons and ions can lose energy through collisions, the electromagnetic energy is dissipated by the collisions and turned into heat [23]. 
The body has a high electric conductivity that results in a large path-loss in the transmission of energy from the implant to free air space. So, our research is focused on implant to implant communication and implant to sub-cutaneous communication channels. The electromagnetic simulations were performed using the $3 \mathrm{D}$ electromagnetic simulation tool Computer Simulation Technology (CST) (https://www.cst.com/) [24]. The voxel data sets mimicking the dielectric properties of human body tissues are used for the calculations of Maxwell equations in the complex medium. The time domain solver of CST has been used and the simulation results are verified with the results from the frequency domain solver of the same software for some cases to validate the consistency of the results.

Each type of voxel representing the biological human tissue will produce its own characteristic response to an imposed electric field [25], which can be mathematically represented with the complex relative permittivity:

$$
\varepsilon(\omega)=\varepsilon+\left(\varepsilon_{s}-\varepsilon_{\infty}\right) /(1+i \omega \tau),
$$

in which $\varepsilon_{\infty}$ is the permittivity measured at a sufficiently high frequency for the voxels (polarizable entities) to be unable to respond to the electric field, $\varepsilon_{s}$ is the limiting low frequency permittivity where the polarisation effect is completely realised, $\omega$ is the angular frequency, $i$ is the complex number $\sqrt{-1}$ and $\tau$ is the characteristic response or relaxation time.

The real and imaginary parts of the relative permittivity can be expressed by

$$
\varepsilon=\varepsilon^{\prime}-i \varepsilon^{\prime \prime},
$$

in which the real part $\varepsilon^{\prime}$ of the permittivity parameter in (2), is given by:

$$
\varepsilon^{\prime}(\omega)=\varepsilon_{\infty}+\left(\varepsilon_{s}-\varepsilon_{\infty}\right) /\left(1+\omega^{2} \tau^{2}\right) .
$$

The imaginary component $\varepsilon^{\prime \prime}$, corresponding to the dissipative loss associated with the polarizable charges moving in phase with the electric field is shown by

$$
\varepsilon^{\prime \prime}(\omega)=\left(\varepsilon_{s}-\varepsilon_{\infty}\right) \omega \tau /\left(1+\omega^{2} \tau^{2}\right) .
$$

The loss factor $\mathcal{E}^{\prime \prime}$ can also be defined in the terms of the frequency-dependent conductivity by

$$
\varepsilon^{\prime \prime}=\frac{\sigma(\omega)}{\omega \varepsilon_{0}}=\left(\sigma_{0}+\sigma_{d}(\omega)\right) / \omega \varepsilon_{0},
$$

where $\sigma_{0}$ is the steady-state conductivity arising mainly from the moving ions and $\sigma_{d}(\omega)$ is the frequency-dependent conductivity arising from dielectric polarization losses.

Our research involved three voxel models: muscle block, Donna and HUGO models. The muscle block model is the simplest one while the HUGO model is the most complex one. The simplified models were used to decrease the computational complexity initially, as multiple simulations in the HUGO model are computationally very expensive. The biological material properties used in the computations are based on 4-
Cole-Cole model provided by Gabriel at the specified frequency $[26,27]$. The formula and parameters used are found under

(http://niremf.ifac.cnr.it/docs/DIELECTRIC/AppendixC.html)

- To use the 4-Cole-Cole model in the calculations, simplifications are needed which is based on curve fitting. Depending on the selected frequency range for broad-band computations, a part of the 4-Cole-Cole model in the given frequency range is generated and curve fitting based on nth order polynomial function is applied for the best fit with minimum least square method.

\section{A. Muscle Block model}

The muscle block model is a very simple electromagnetic model with resolution of $1 \mathrm{~mm} \times 1 \mathrm{~mm} \times 1 \mathrm{~mm}$. The two communication elements, the capsules, are modeled as simple dipole antennas with a length of $5 \mathrm{~mm}$ and a diameter of $2 \mathrm{~mm}$ enclosed on all sides in a vacuum tube of width $1 \mathrm{~mm}$. The vacuum gap of $1 \mathrm{~mm}$ is provided on all sides to prevent the direct contact of the antenna with the body tissues. To classify the frequency which produces the least path-loss between the two capsules, a $500 \mathrm{~mm} \times 500 \mathrm{~mm} \times 500 \mathrm{~mm}$ cubic block is used, with its permittivity representing that of the human muscle (see Fig. 2). The muscle block contains a centrally located sphere of diameter $50 \mathrm{~mm}$ with permittivity representing that of human blood. This model is designed for a simple representation of one of the four chambers of the human heart. The permittivity of both the muscle block and the blood sphere changes according to the frequencies since we know that the permittivity of human tissues are frequency dependent. Two simple, fixed-length dipoles are placed at two ends of the sphere and the path-loss is calculated using the scattering parameter $\left(S_{21}\right)$ of the antenna when a Gaussian pulse of power $1 \mathrm{~W}$ is transmitted. The distance between the dipoles is fixed at $50 \mathrm{~mm}$ and the frequency range of $300 \mathrm{MHz}$ to $3 \mathrm{GHz}$ is analyzed.

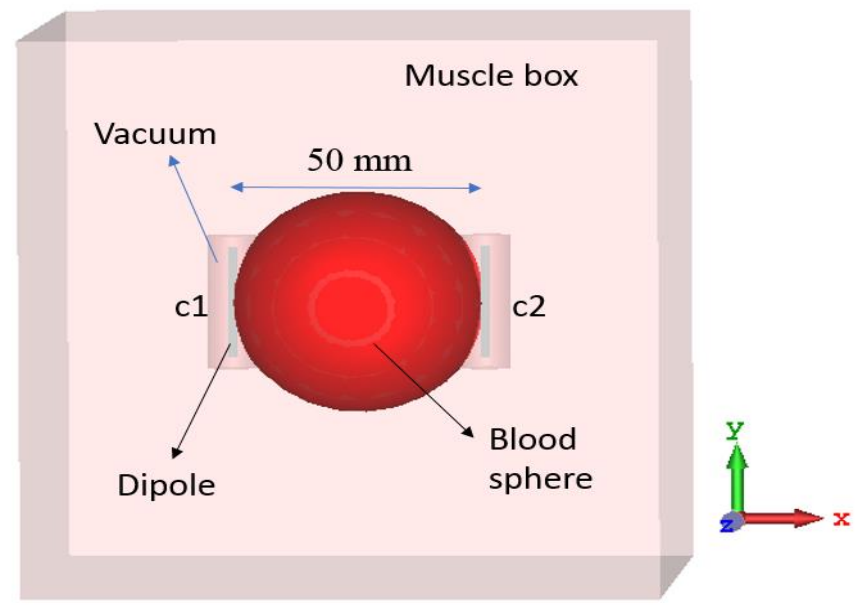

Fig. 2 Dipoles c1 and c2 enclosed in vacuum placed at two ends of the diameter of a sphere containing blood inside a muscle block. The resolution of each voxel is $1 \mathrm{~mm} \times 1 \mathrm{~mm} \times 1 \mathrm{~mm}$ and the model has been simulated in CST in a frequency range of $0.3-3 \mathrm{GHz}$. The size of the blood volume has been reduced in this figure for better visual representation of the dipole antennas

A quadratic equation of order two is used to fit the data which represents the variations of permittivity and conductivity with 
frequency. A linear function could simplify the simulations and decrease the computational time but it could not perfectly fit the data. So, a quadratic function is used instead of a linear one.

The number of dielectric data samples taken for the quadratic fitting is fixed to 100 in the entire frequency range between 0.3 $-3 \mathrm{GHz}$. The maximum error limit between the real data and the quadratic fitting is fixed to 0.01 . A curve-fitting algorithm is used to find the best fit. It is observed that the quadratic equation of order 2 could best fit the frequency dependent dielectric curve (see Eq. 2-5). Decreasing the maximum error limit and increasing the number of the data points could provide better curve fitting but this would highly increase the computational time. Dielectric properties of both blood and muscle vary depending on the frequency (see Fig. 3).

\section{B. Donna model}

The Donna model is a biological model from the CST Voxel family [28]. It is a heterogeneous body model of a middle-aged oversized 40 years old woman with a weight of 79 kilograms.

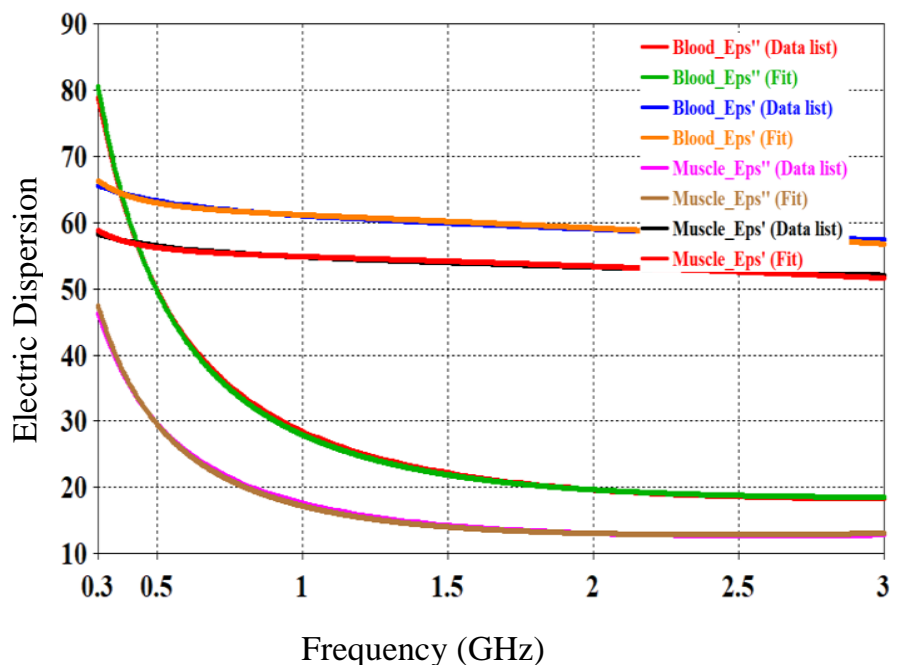

Fig. 3 Dielectric properties of human blood and muscle in frequency range of $0.3-3 \mathrm{GHz}[29]$

The resolution of the model is $1.875 \mathrm{~mm} \times 1.875 \mathrm{~mm} \times 10 \mathrm{~mm}$. Though it is a heterogeneous model, the heart is represented as a homogeneous volume composed of voxels with average permittivity and conductivity of human heart tissues. The permittivity and the conductivity of the heart corresponding to the frequency is given in fig. 4 . A quadratic function of order 2 is used to fit the data which can be used for simulations. The Cole-Cole parameters for the modelled biological tissues are provided in Table I.

\section{TABLE I}

COLE- COLE PARAMETERS FOR THE MODELLED BIOLOGICAL TISSUES [29]

\begin{tabular}{l|c|c|c}
\hline Tissues & $\varepsilon_{\infty}$ & $\varepsilon_{s}$ & $\tau(\mathrm{ps})$ \\
\hline Blood & 4 & 92 & 8.33 \\
Muscle & 4 & 54 & 7.23 \\
Heart & 4 & 61 & 8.05 \\
\hline
\end{tabular}

The dipole antennas of length $5 \mathrm{~mm}$ and diameter $2 \mathrm{~mm}$ are placed at two positions inside the heart to create a communication link between them. One of the dipole is placed inside the right ventricle close to the apex of the heart and another inside the right atrium (see Fig. 5a). The upper torso of the Donna model starting from the lower base of the neck to the upper abdomen is used for simulation. The full-body simulation is not done since it is computationally expensive and is not required for the estimation of the channel models inside- or close to the heart.

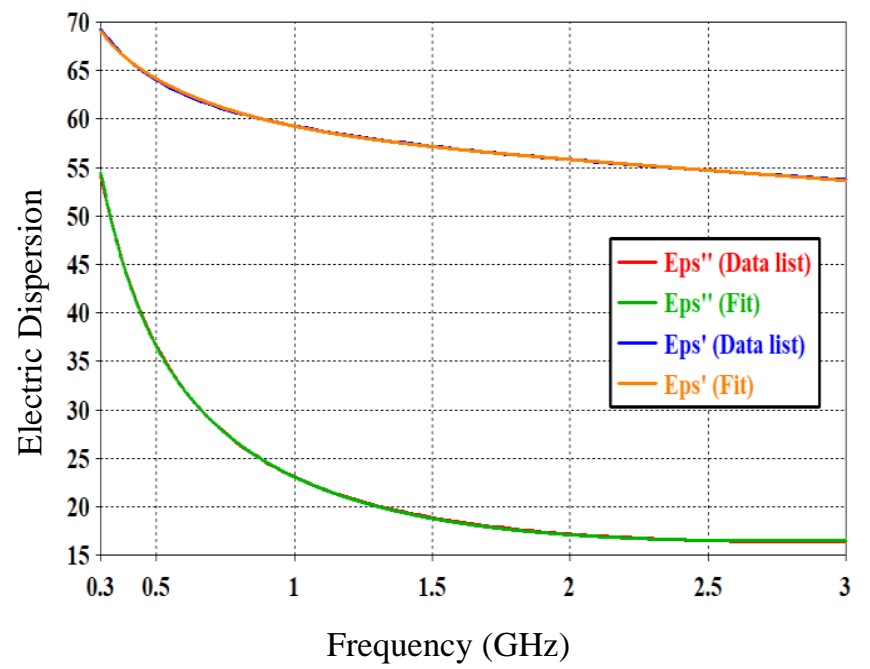

Fig. 4 Dielectric properties of human heart in Donna model in frequency range of $0.3-3 \mathrm{GHz}$ [29]

Another simulation is done on the same model, but with changing the location of the right atrium dipole to a subcutaneous position on the upper-chest where the can (containing the electronics and lead connections) of a pacemaker is normally placed (see Fig. 5b).

These simulations help us to get an idea of the signal loss at different frequencies and choose the appropriate frequency for the multi-node capsule pacemaker technology.

\section{HUGO model}

The anatomical data set used in our research is of the Visible Human Project [30, 31] and the voxel model developed from it in CST is known as HUGO model. The model is based on a dissected male corpse sliced into several thousand layers, which are sampled and interpolated together, creating a highly detailed computational model. The import is performed via an interface that allows different resolutions $(1 \mathrm{~mm}-8 \mathrm{~mm})$ and different material tissues to be selected. The resolution of $1 \mathrm{~mm}$ by $1 \mathrm{~mm}$ is used for the simulations in this study. The area of interest is selected in a side view and front view manner (see Fig. 6).

The model is very detailed and considers the dielectric properties of each biological material separately. The dielectric properties of individual types of human body tissues are modelled to mimic the changes in the permittivity and conductivity values with respect to frequency. The upper torso of the HUGO model starting from the lower base of the neck to the upper abdomen is used for simulation. Like the Donna model, the full-body simulation is not done in the HUGO model also since it is computationally expensive and not required for 


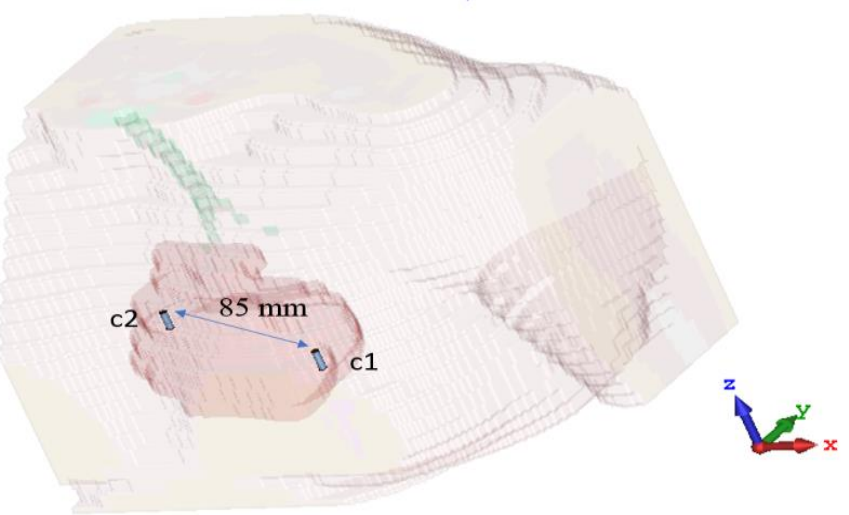

(a)

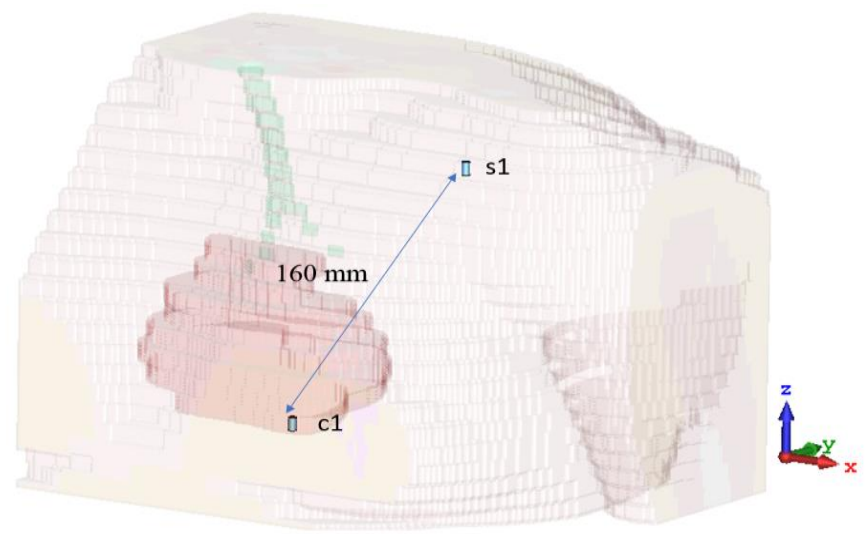

(b)

Fig. 5 (a) Dipole antenna c1 placed inside the right ventricle close to the apex of the heart and c2 inside the right atrium in Donna voxel model

(b) Dipole antenna c1 placed inside the right ventricle close to the apex and s1 subcutaneously placed on the shoulder where the can of a pacemaker is normally placed.

the estimation of the channel models inside- and close to the heart.

The dielectric property curves of two main types of tissues, blood and muscle, for the entire frequency range, were provided previously (see Fig. 3). This model included several other tissue-types such as fat, skin, bone, cartilages, bone marrow, lung etc.

The motivation is to build a communication model between three capsules placed inside the heart. The proposed positions of the three capsules inside the heart is in the right ventricle close to the apex of the heart, right atrium, and left ventricle (see Fig. 6a). The other motivation is to find the optimum position of the sub-cutaneous implant for which the capsules are placed subcutaneously in the shoulder, lateral side of the body and abdomen wall (see Fig. 6b).

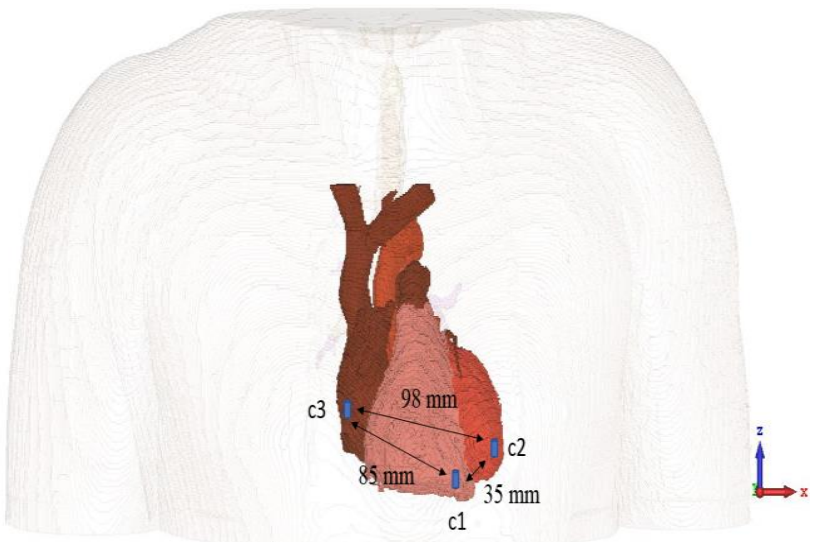

(a)

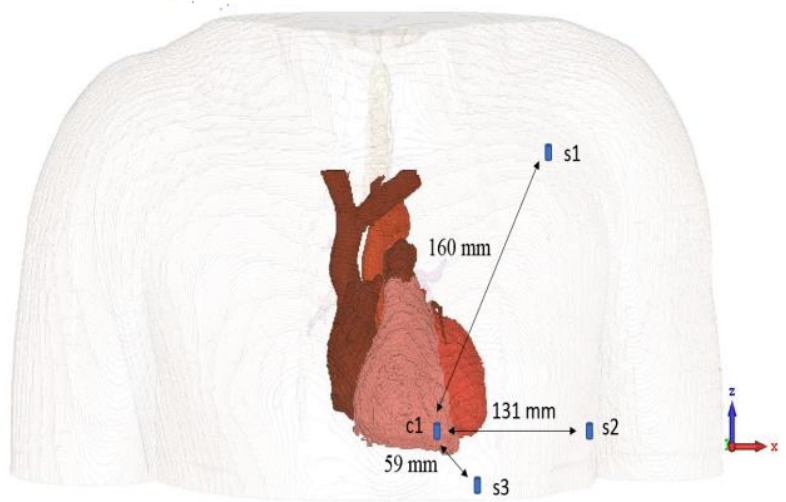

(b)

Fig. 6 Dipoles placed at different positions in HUGO model in CST a) implant to implant communication inside the heart $b$ ) heart to sub-cutaneous implant communication.

During heartbeat, the volume of the blood inside the heart chambers constantly vary, between end-diastole when the heart is dilated with maximum blood volume and end-systole where the heart is contracted and contains minimum volume. Due to the high mineral content, blood is a highly dispersive medium. The blood volume in the right ventricle approximately varies between $14 \mathrm{~mL}$ to $180 \mathrm{~mL}$ for a human heart between endsystole and end-diastole [32]. To simulate how the variation of blood volume affects the signal transmission, we designed a simulation scenario using the same simulation tool as in the previous section. A blood-containing sphere is placed inside the right ventricle of the heart of the HUGO model and the diameter of the sphere is varied in proportion to the ventricular blood flow (see Fig. 7). The simulation is run for sphere radiuses of $15,20,25$ and $35 \mathrm{~mm}$ which accounts for 14, 33, 65 and 180 $\mathrm{mL}$ of blood respectively. Capsule $\mathrm{C} 1$ is placed in the right ventricle, $\mathrm{C} 2$ in the left ventricle and $\mathrm{S} 1$ in the shoulder subcutaneously. The simulations are performed to investigate the change in the received signal power at $2.4 \mathrm{GHz}$ during the cardiac cycle. 


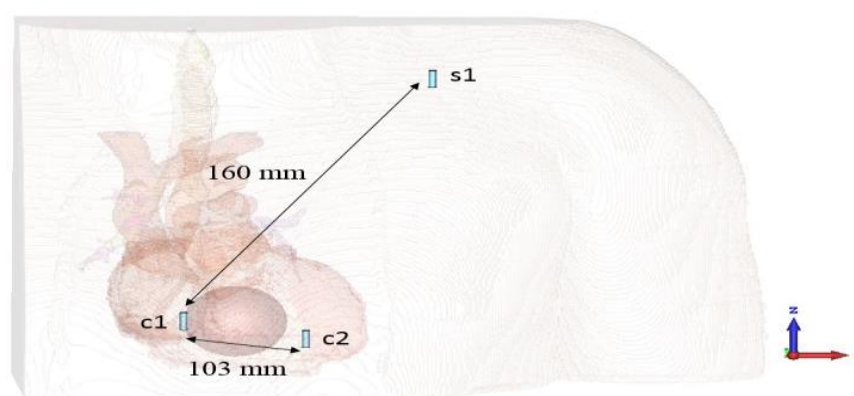

Fig 7. Capsules are placed in the right ventricle $(\mathrm{C} 1)$, left ventricle $(\mathrm{C} 2)$ and shoulder (S1). A sphere containing blood is placed in the right ventricle whose volume is changed to simulate the different ventricular blood volumes during the cardiac cycle

The dipole antennas are always oriented along the z-axis which is in the coronal plane [33] of the human body. The normalization of the S-parameters is done to remove the mismatch effects of the transmitter antenna (Tx) and receiver antenna ( $\mathrm{Rx})$ and to find the coupling between the antennas [34]. The normalization of the power received by Rx from Tx is calculated using the following equation

$$
\left|S_{21}\right|_{\text {norm }}=\frac{\left|S_{21}\right|}{\sqrt{1-\left|S_{11}\right|^{2}} \sqrt{1-\left|S_{22}\right|^{2}}}
$$

where $\left|S_{21}\right|_{\text {norm }}$ is the normalized power received, $S_{21}$ is the power received before normalization, $S_{11} \& S_{22}$ are the return losses of the Tx and Rx respectively.

The Tx mismatch effect is removed using the following equation

$$
\left|S_{11}\right|_{\text {norm }}=\frac{\left|S_{11}\right|}{1-\left|S_{11}\right|^{2}}
$$

where $\left|S_{11}\right|_{\text {norm }}$ is the normalized return loss of the Tx and $\left|S_{11}\right|$ is the return loss before normalization.

The normalization of the S-parameters are done using mathematical techniques as it is impossible to match the antenna over such wide frequency range using the matching networks.

\section{Results}

The results from different bio-models after simulation in CST are recorded over the entire-frequency range from 0.3 to 3 GHz.

\section{A. Muscle block results}

Because of reciprocity, interchanging $\mathrm{Tx}$ and $\mathrm{Rx}$ will not affect the outcome. The coupling varies between $-130 \mathrm{~dB}$ to $-84.5 \mathrm{~dB}$ in the frequency range of $0.3 \mathrm{GHz}$ to $3 \mathrm{GHz}$ (see Fig. 8). The lowest coupling of $-130 \mathrm{~dB}$ is see at $738 \mathrm{MHz}$ whereas the highest coupling of $-84.5 \mathrm{~dB}$ is observed at $2.73 \mathrm{GHz}$. The result shows that the coupling approaches maximum in the ISM band. The coupling varies between $-89 \mathrm{~dB}$ and $-87 \mathrm{~dB}$ in the frequency range of $2.4 \mathrm{GHz}$ to $2.5 \mathrm{GHz}$. In the muscle block model, the signal mainly passes through the blood, which is highly lossy material. Consequently, the attenuation of the signal is higher in muscle block model than in normal human

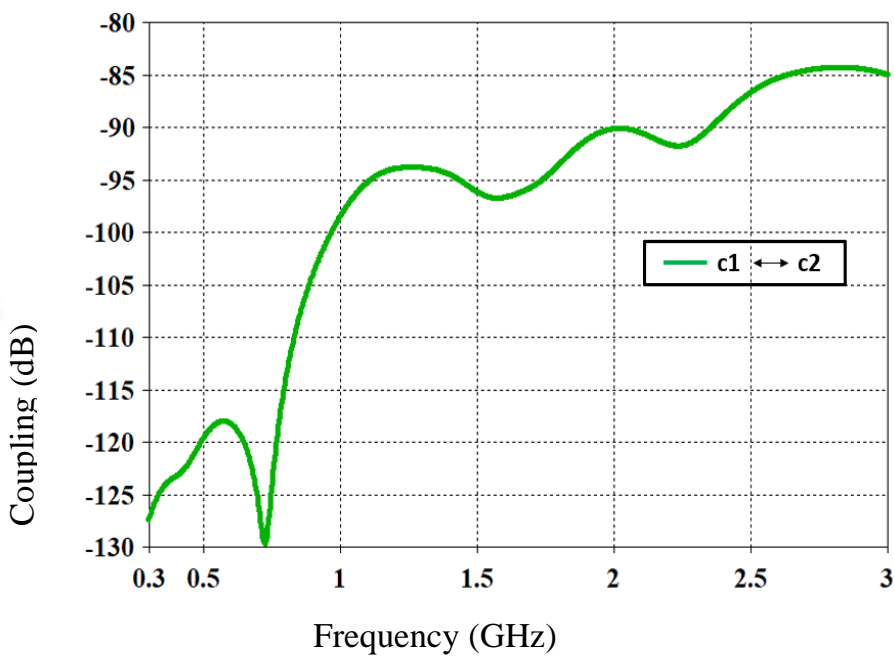

Fig. 8 Coupling in $\mathrm{dB}$ between the Tx and Rx in frequency range of $0.3 \mathrm{GHz}$ to $3 \mathrm{GHz}$ in the muscle block model. The capsules $(\mathrm{C} 1, \mathrm{C} 2)$ are placed in the muscle on the either ends of the diameter of a sphere containing blood.

heart which does not only contain muscle and blood, but also less attenuating mediums, such as fat and other connective tissues.

\section{B. Donna model results}

The simulations in Donna model (see Fig. 9 and 10) shows the coupling between the capsules placed in the left ventricle and subcutaneously in the shoulder respectively, when the signal is transmitted from the capsule placed in the right ventricle. In the simulations of cardiac implant to cardiac implant, the coupling fluctuates between $-133.5 \mathrm{~dB}$ and -88.5 $\mathrm{dB}$ in the frequency range of $0.3 \mathrm{GHz}$ to $3 \mathrm{GHz}$. The least coupling is at $2.09 \mathrm{GHz}$ whereas the highest is at $1.18 \mathrm{GHz}$ and varies between $-93.7 \mathrm{~dB}$ and $-89.6 \mathrm{~dB}$ in the ISM frequency band.

In the simulation of cardiac implant to subcutaneous implant in shoulder, the coupling varies between $-133.1 \mathrm{~dB}$ and -95.1 $\mathrm{dB}$ in the frequency range of $0.3 \mathrm{GHz}$ to $3 \mathrm{GHz}$. The least coupling is noticed at $2.67 \mathrm{GHz}$ whereas the highest is at 2.23 GHz. It varies from $-98.4 \mathrm{~dB}$ to $-96.2 \mathrm{~dB}$ in the ISM band.

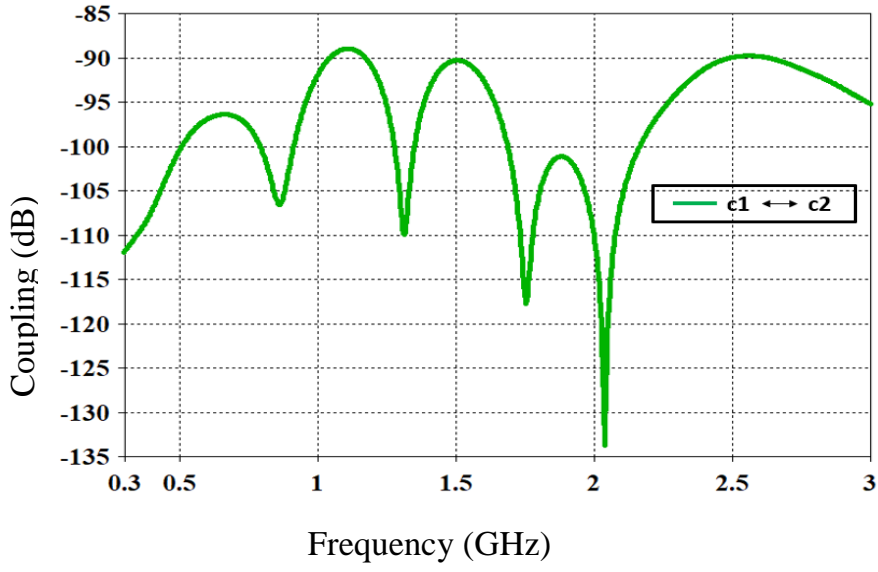

Fig. 9 Coupling in $\mathrm{dB}$ between the receiver antenna $(\mathrm{C} 2$ is placed in the left ventricle of the heart) and the transmitter antenna ( $\mathrm{C} 1$ is placed in the right ventricle of the heart close to the apex) in frequency range of $0.3 \mathrm{GHz}$ to 3 $\mathrm{GHz}$ in the Donna model 


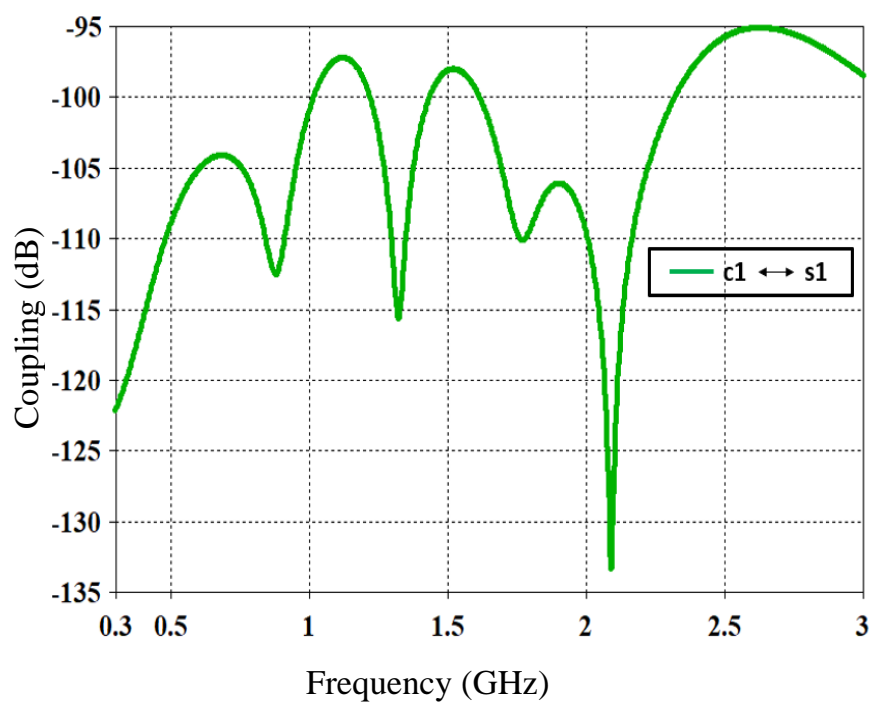

Fig. 10 Coupling in $\mathrm{dB}$ between the receiver antenna ( $\mathrm{S} 1$ is placed in the shoulder subcutaneously) and the transmitter antenna ( $\mathrm{C} 1$ is placed in the right ventricle of the heart close to the apex) in frequency range of $0.3 \mathrm{GHz}$ to 3 $\mathrm{GHz}$ in the Donna model

The Donna model gives approximate results because the heart is modelled as a homogeneous medium with dielectric properties of all heart tissues averaged. The simulations on the more sophisticated HUGO model provide more realistic results. Moreover, very large fading variations can be noticed in the coupling at some frequencies. This is due to the uneven voxel size of the Donna model. Each voxel has a length and breadth of $1.875 \mathrm{~mm} * 1.875 \mathrm{~mm}$ but a height of $10 \mathrm{~mm}$ which is nearly six times of that of the length and breadth. This causes sudden impedance mismatch in the wave propagation. This is also the reason behind the minimum points in Figs. 8, 9 and 10. This can be avoided in the HUGO model as it has equal dimensions on all sides of $1 \mathrm{~mm} * 1 \mathrm{~mm} * 1 \mathrm{~mm}$. In addition, as the frequency of simulations is at $2.4 \mathrm{GHz}$, the wavelength in the biological medium is about $17 \mathrm{~mm}$ (for $\varepsilon^{\prime}=50$ ). Therefore, some voxels are in the range of $\lambda / 2$ or more. Therefore, accurate results can be achieved using Hugo model. The other models would be appropriate for lower frequencies. Donna model shows uneven characteristics at higher frequencies compared to lower frequencies below $700 \mathrm{MHz}$ as seen from the simulation results. So, the available voxel resolution of the Donna model is not appropriate for conducting experiments at higher frequencies.

\section{HUGO model results}

The simulations in the HUGO model is done for the entire frequency range of $0.3 \mathrm{GHz}$ to $3 \mathrm{GHz}$ for three-capsule cardiac pacemaker system (see fig. 6a). The results show that the coupling varies between $-101.2 \mathrm{~dB}$ and $-64.9 \mathrm{~dB},-86.8 \mathrm{~dB}$ to $71.1 \mathrm{~dB}$ and $-109.8 \mathrm{~dB}$ to $-76.1 \mathrm{~dB}$ for $\mathrm{C} 1$ to $\mathrm{C} 2, \mathrm{C} 1$ to $\mathrm{C} 3$ and $\mathrm{C} 2$ to $\mathrm{C} 3$ pacemaker capsule-configuration respectively (see Fig. 11). For the $\mathrm{C} 1$ to $\mathrm{C} 2$ pacemaker configuration, the maximum coupling of $-64.9 \mathrm{~dB}$ is seen at $2.42 \mathrm{GHz}$ which lies within the ISM frequency band. For the $\mathrm{C} 1$ to $\mathrm{C} 3$ and $\mathrm{C} 2$ to $\mathrm{C} 3$ capsule configurations, the coupling in the ISM band is also close to its maximum. The results show that the variation of coupling with frequency is smooth and less fading is visible due to better voxel resolution in comparison to the Donna model.

Another objective of the paper is to define the optimal placement site for the small-sized subcutaneous implant. To investigate various potential sites, the simulations are done by placing the capsule in the shoulder, lateral side of the body and in the abdominal wall (see Fig. 6b). The results show that the coupling varies from $-101.9 \mathrm{~dB}$ to $-79.8 \mathrm{~dB},-129.7 \mathrm{~dB}$ to -97.1 $\mathrm{dB}$ and $-142.4 \mathrm{~dB}$ to $-116.9 \mathrm{~dB}$ for $\mathrm{C} 1$ to $\mathrm{S} 3, \mathrm{C} 1$ to $\mathrm{S} 2$ and $\mathrm{C} 1$ to $\mathrm{S} 1$ capsules configuration respectively (see Fig. 12). The maximum coupling of $-79.8 \mathrm{~dB}$ is observed for the $\mathrm{C} 1$ to $\mathrm{S} 3$ capsule configuration at the frequency of $2.39 \mathrm{GHz}$. For the other configurations, the coupling approaches maximum in the ISM band but it is rather less compared to the $\mathrm{C} 1$ to $\mathrm{S} 3$ configuration. This shows that the outer wall of the abdomen is a better position for the placement of the sub-cutaneous implant as compared to shoulder and lateral side of the body. Since, the size of the implant is very small, the effort in placing the implant to the abdomen wall will be nominal without causing much discomfort to the patients as suggested by the medical doctors.

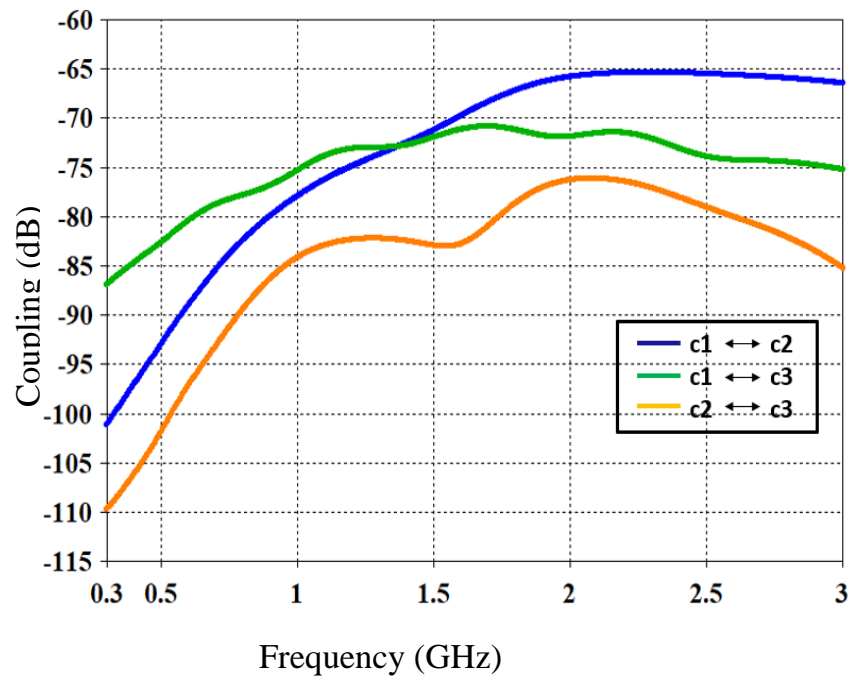

Fig. 11 Coupling in $\mathrm{dB}$ between the transmitter and receiver antennas in frequency range of $0.3 \mathrm{GHz}$ to $3 \mathrm{GHz}$ in the HUGO model. The capsule $\mathrm{C} 1$ is placed in the right ventricle close to the apex, $\mathrm{C} 2$ is placed in the left ventricle and $\mathrm{C} 3$ is placed in the right atrium of the heart 


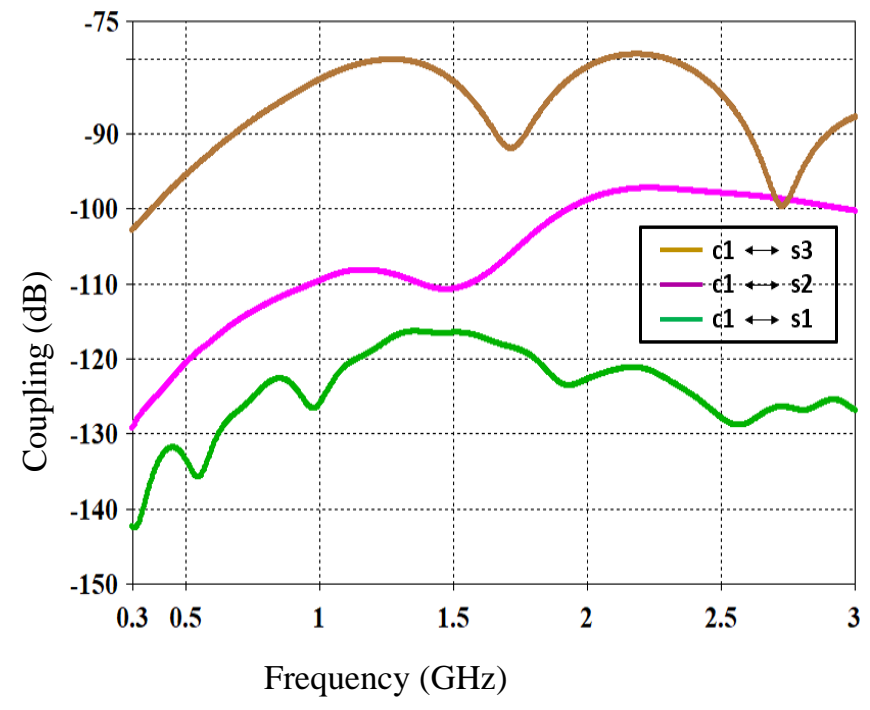

Fig. 12 Coupling in $\mathrm{dB}$ between the transmitter and receiver antennas in frequency range of $0.3 \mathrm{GHz}$ to $3 \mathrm{GHz}$ in the $\mathrm{HUGO}$ model. The capsule $\mathrm{C} 1$ is placed in the right ventricle of the heart close to the apex, S1 is subcutaneously placed in the shoulder, S2 in the lateral side of the body and $\mathrm{S} 3$ on the abdomen wall

coupling (see Table II). At $2.4 \mathrm{GHz}$, the coupling varies by 3.1 $\mathrm{dB}$ for the $\mathrm{C} 1$ to $\mathrm{C} 2$ configuration and by $1.3 \mathrm{~dB}$ for the $\mathrm{C} 1$ to $\mathrm{S} 1$ configuration between $14 \mathrm{~mL}$ and $180 \mathrm{~mL}$ spheres of blood. The results confirm that the coupling decreases with the increase in cardiac blood volume. The variation is coupling is not too high because the distance between the capsules is kept constant. When the blood volume is increased, the voxels representing muscle is converted to voxel representing blood. Whereas, in real scenario the capsules would move with cardiac movements during the cardiac cycle resulting in different distances between them. This would further cause significant variation in the coupling in different scenarios. The dynamic movement of the heart could not be simulated in our model as it can only simulate static conditions.

\section{TABLE II}

COUPLING IN dB BETWEEN THE TRANSMITTER AND RECEIVER ANTENNAS AT 2.4 GHZ IN THE HUGO MODEL WITH CHANGE IN VENTRICULAR BLOOD VOLUME. A) THE TRANSMITTER CAPSULE C1 IS PLACED IN THE RIGHT VENTRICLE CLOSE TO THE APEX AND RECEIVER CAPSULE C2 IS PLACED IN THE LEFT VENTRICLE

B) THE TRANSMITTER CAPSULE C1 IS PLACED IN THE RIGHT VENTRICLE CLOSE TO THE APEX AND RECEIVER CAPSULE S1 IS PLACED IN THE SHOULDER SUBCUTANEOUSLY.

\begin{tabular}{c|c|c|c|c}
\hline Blood Volume $(\mathrm{mL})$ & 14 & 33 & 65 & 180 \\
\hline $\mathrm{A}: \mathrm{C} 1-\mathrm{C} 2(\mathrm{~dB})$ & -81.8 & -82.7 & -83.1 & -84.9 \\
$\mathrm{~B}: \mathrm{C} 1-\mathrm{S} 1(\mathrm{~dB})$ & -127.6 & -127.9 & -128.7 & -128.9 \\
\hline
\end{tabular}

\section{LIVING ANIMAL EXPERIMENTAL SETUP}

The results from the mathematical simulation of the cardiac implant to sub-cutaneous implant communication are validated by an in-vivo experiment on an adult pig. A simple transmitter capsule is designed which is a plastic container containing a printed circuit board (PCB), an antenna and a battery (see Fig 14a). The PCB contains a voltage-controlled oscillator (VCO) IC (HMC385LP4E) operating at $3 \mathrm{~V}$ and generating sinusoidal signal at $2.4 \mathrm{GHz}$. The antenna is $1 \mathrm{~mm}$ radius meander shaped with a resonant frequency of $2.4 \mathrm{GHz}$ which is matched at that frequency. The antenna used in the experiments is a scaled version of our implant antenna published in [35]. The measured S11 inside the plastic container within a liquid phantom, including an air gap around the antenna, is less than $-8 \mathrm{~dB}$ at 2.4 $\mathrm{GHz}$. We note that the measurement of such small antennas using cables cannot be very accurate, but we estimate that the antenna is well matched to the source impedance. The subcutaneous implant antenna (Fig. 14b) is a wide-band patch antenna of dimensions $3 \mathrm{~cm} * 3 \mathrm{~cm}$ which is the receiver antenna and has a wideband impedance matching with $\mathrm{S} 11<$ $11 \mathrm{~dB}$. The antenna is encapsulated using plastic covering to prevent direct contact with tissues.

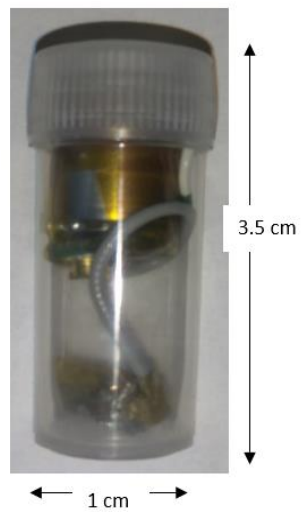

(a)

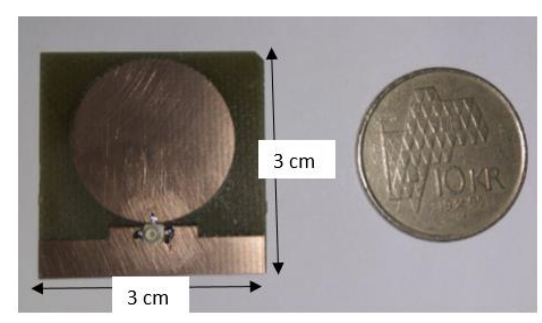

(b)
Fig. 14 a) The transmitter implant capsule containg the PCB, battery and meander antenna b) sub-cutaneous implant patch antenna

The in-vivo animal experiments are performed on a pig weighing $61 \mathrm{kgs}$ (see Fig. 15). To evaluate and compare the performances of the capsule in an environment similar to that of the human body, the pig is prepared under a general anesthetic and used in this experiment. All studies are performed at the hybrid operating room of the Intervention Centre, Rikshospitalet, Oslo University Hospital, Norway that has accreditation for conducting animal experiments. All the experiments are performed in strict compliance with laws regarding Ethical standards and humane treatment of animals. Because this animal was alive and all of its organs were operating normally, the experimental conditions were virtually identical to the conditions expected in a human body. The implant transmitter capsule is placed behind the right ventricle by making making an incision on the chest of the pig. The incisions are closed to prevent any leakage of signal through the holes (see Fig. 16). The sub-cutaneous implant is placed under the skin near the shoulder. The antenna is connected to the spectrum analyser via the probes. The received power is directly visualised using a spectrum analyser and saved for post-processing. The sub-cutaneous implant is placed at other positions like the lateral side of the body and wall of the 


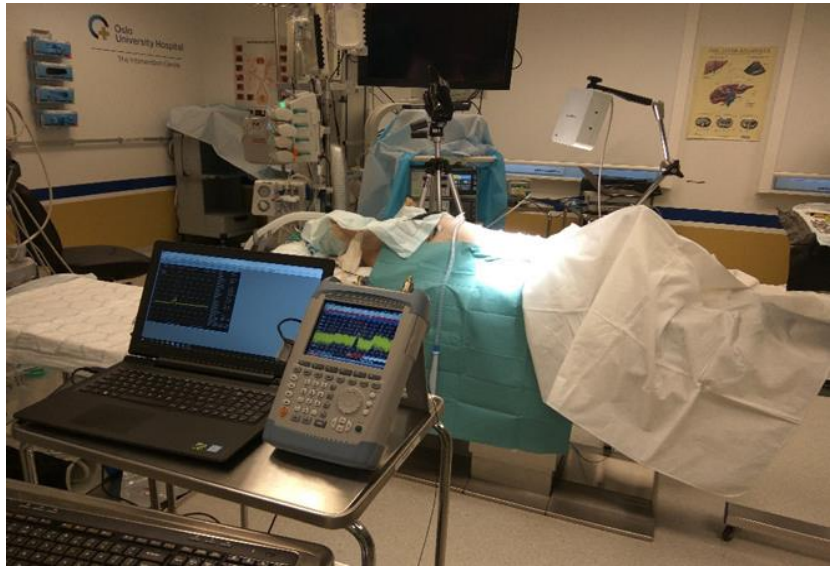

Fig. 15 Experimental setup for the animal experiment in Intervention Centre, Oslo University Hospital in Norway on Oct 12, 2017

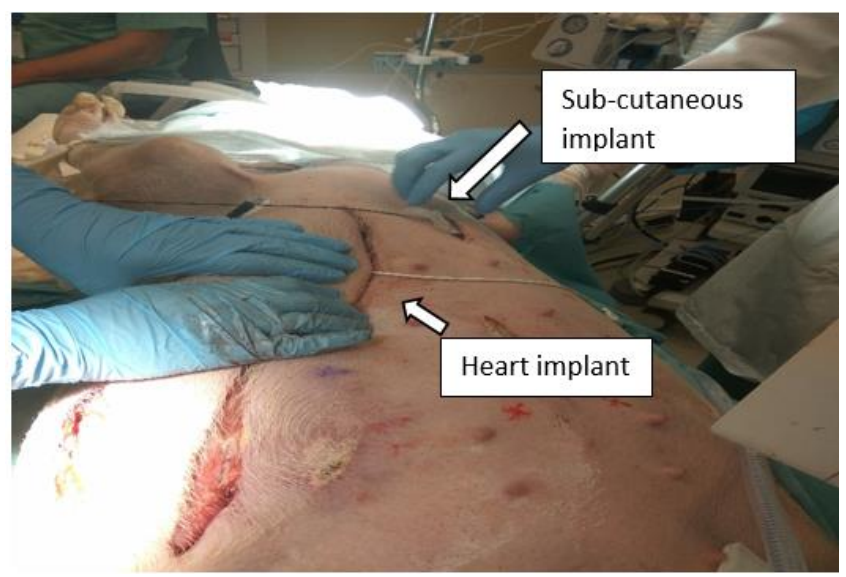

Fig 16. Photograph showing the implant antenna and the sub-cutaneous implant antenna

abdomen to find the optimal placement of the sub-cutaneous implant. The distance between the implants are measured with the help of an electromagnetic distance measurement system called Medical Aurora manufactured by the company northern Digital Inc. that gives accurate distance measurements.

The living animal experiment results is in good accordance with the simulation results. Living animal experiments are used to obtain the coupling at few discrete points. Since, it is difficult to maneuver the implants inside the pig to obtain multiple desirable distances to obtain a complete path-loss model, few recording are done to compare with the simulation results .The coupling is $-88.31 \mathrm{~dB}$ at a distance of $130 \mathrm{~mm}$ between the cardiac implant and the sub-cutaneous implant on the shoulder (see Table III). The coupling improves by $4.09 \mathrm{~dB}$ when the sub-cutaneous cutaneous implant is placed on the lateral side of the body at the same distance of $130 \mathrm{~mm}$ between the two implants. The highest coupling is seen when the sub-cutaneous implant is placed on the outer wall of the abdomen. A coupling of $-75.2 \mathrm{~dB}$ is obtained at a distance of $100 \mathrm{~mm}$ between the two implants. This coupling is better than that of the lateral side of the body and shoulder by $9.02 \mathrm{~dB}$ and $13.11 \mathrm{~dB}$ respectively. This improvement is due to the proximity of the implant to the heart as well as lower losses in the bio-logical tissues in- between the heart implant and the sub-cutaneous implant placed in the abdominal wall. Thus, the animal experiment results confirm the simulation results that the abdominal wall is the optimal position for the placement of the implant compared to shoulder and lateral side of the body.

\section{TABLE III}

COUPLING IN Db BETWEEN THE TRANSMITTER AND RECEIVER ANTENNAS PLACED AT DIFFERENT POSITIONS OF THE ANIMAL AT THE OPERATING FREQUENCY OF $2.4 \mathrm{GHz}$

\begin{tabular}{|c|c|c|c|}
\hline $\begin{array}{l}\text { Transmitter } \\
\text { Implant } \\
\text { position } \\
\end{array}$ & $\begin{array}{l}\text { Receiver } \\
\text { Implant } \\
\text { position }\end{array}$ & $\begin{array}{l}\text { Distance } \\
(\mathrm{mm})\end{array}$ & $\begin{array}{l}\text { Coupling } \\
\text { (dB) }\end{array}$ \\
\hline Right ventricle & Shoulder & 130 & -88.31 \\
\hline Right ventricle & Lateral side & 130 & -84.22 \\
\hline Right ventricle & $\begin{array}{l}\text { Outer wall of } \\
\text { abdomen }\end{array}$ & 100 & -75.2 \\
\hline
\end{tabular}

The simulations are based on human model and the experiments are on an animal. The size and shape of the models are not comparable. However, the objective of the study is not exactly to correlate the simulation results with the animal experiment results but to find the optimal placement of the subcutaneous implant. Both the numerical simulations and animal experiments proves it which is quite encouraging. The results cannot be directly correlated due to different contributing factors. The simulation considers an ideal environment whereas the experimental setup involves interference from surrounding environment. An ideal dipole antenna is used for the numerical simulations whereas in case of the animal experiments, a meander-shaped antenna of a different shape is used for the heart implant and a patch antenna for the receiver implant to collect the received signal. The other contributing factors are impedance mismatch of the antennas, antenna rotation, polarization mismatch and misalignment of the antenna in the animal experiment. These different contributing factors results in a significant difference between the results obtained from the software simulations and the animal experiments.

The results from all the bio-models show that the ISM band is the best frequency range for the operation of our given size implant antennas in the cardiac application. The normalized antenna size and radiation efficiency increase with frequency so the antenna performance increases at higher frequencies. Alternatively, the material losses also increase with the increase in frequency. The overall implant antenna efficiency is a result of the combination between the normalized size of the antenna and the material losses. Consequently, the best performance is observed in the ISM band with the dipole antenna of length 5 $\mathrm{mm}$ and diameter $2 \mathrm{~mm}$.

In conventional pacemakers, one of the motivation behind placing the pacemaker can in a subcutaneous pocket in the shoulder is the large size of the can, which houses all electronics, whereas the battery occupies most of the volume. Placing such large cans in the lateral side of the body or abdominal wall would not only cause discomfort to the patients, but also have higher complication risks due to the thinner skin 
and greater plasticity of the abdomen. One of the main objectives of our project is to build a small-sized capsule based on novel power optimization strategies, such as distributing the power requirements between several nodes, which make up a multi-nodal pacemaker system. The small capsules may be placed in different locations within the body, without the constraints of the patient discomfort and can-related complication risks. Both the numerical analysis and animal experiments show that the abdominal wall is the optimal position for the placement of the implant in comparison to the shoulder and the lateral abdomen. This is due to the proximity of the implant to the heart, where the other antenna is implanted, as well as the lower losses in the biological tissues in-between the two implants.

\section{CONCLUSIONS AND FUTURE WORK}

In this paper, the propagation of RF signals for in-body to inbody implants have been analyzed for future cardiac pacemaker applications. The analysis is not done by averaging all the body tissues but rather involving the influences of all tissue layers between the implants, including the specific shape and size of them. The optimal position of the sub-cutaneous implant is determined based on the simulation results and living animal experiments.

We concluded in this paper that the ISM radio band of the frequency range of $2.4-2.5 \mathrm{GHz}$ is optimal for the multi-node pacemaker technology due to the size constraints of the capsule antenna. Since the implant path loss is a combination of the path loss due to the biological tissues with the antenna efficiency and the length of the implant antenna is much smaller compared to the wavelength in the frequency range of $0.3-3 \mathrm{GHz}$, the overall antenna efficiency is higher at higher frequencies. This results in better antenna performance in the ISM band.

The next goal is to validate these simulation results with experimental studies on simple phantom models. The phantoms models are simple sucrose solutions with some other chemicals that mimics the permittivity of human tissues at different frequencies. More in-vivo experiments will be done on animals by placing the capsules at different positions and collecting the signals between the implants using probes. The simulation results provide a rational basis before more animal experiments, help better planning and possibly reduce the number of such experiments.

\section{ACKNOWLEDGMENT}

This work was funded by the European Union's H2020: MSCA: ITN program for the "Wireless In-body Environment Communication - WiBEC" project under the grant agreement no. 675353 .

\section{REFERENCES}

[1] S. D. Goedeke, J.G. Keimel and D.L. Thompson, "Adaptive, performanceoptimizing communication system for communicating with an implanted medical device," U.S. Patent No. 5,843,139, Dec. 1998.

[2] K. Y. Yazdandoost, K. Sayrafian-pour and K. Hamaguchi, "RF Propagation and Channel Modeling for UWB Wearable Devices", IEICE Transactions on Communications, vol. 94-, no. 5, pp. 1126-1134, May 2011.
[3] K. Sayrafian-Pour, W.-B. Yang, J. Hagedorn, J. Terrill, and K. Y. Yazdandoost, "A statistical path loss model for medical implant communication channels," IEEE 20th International Symposium on Personal, Indoor and Mobile Radio Communications, pp. 2995-2999. Sept. 2009.

[4] S. L. Cotton, R. Derrico, and C. Oestges, "A review of radio channel models for body centric communications," Radio Science, vol. 49, no. 6, pp. 371-388, Jun 2014.

[5] M. Chen, S. Gonzalez, A. Vasilakos, H. Cao, and V. C. M. Leung, "Body Area Networks: A Survey," Mobile Networks and Applications, vol. 16, no. 2, pp. 171-193, Apr. 2010.

[6] D. B. Smith, D. Miniutti, T. A. Lamahewa, and L. W. Hanlen, "Propagation Models for Body-Area Networks: A Survey and New Outlook," IEEE Antennas and Propagation Magazine, vol. 55, no. 5, pp. 97-117, Oct. 2013.

[7] P. D. Bradley, "An ultra low power, high performance Medical Implant Communication System (MICS) transceiver for implantable devices," IEEE Biomedical Circuits and Systems Conference, pp. 158-161, Nov. 2006.

[8] M. R. Yuce, S. W. P. Ng, N. L. Myo, J. Y. Khan, and W. Liu, "Wireless Body Sensor Network Using Medical Implant Band," Journal of Medical Systems, vol. 31, no. 6, pp. 467-474, Jan. 2007.

[9] M. R. Yuce, S. W. P. Ng, N. L. Myo, C. K. Lee, J. Y. Khan, and W. Liu, "A MICS Band Wireless Body Sensor Network," IEEE Wireless Communications and Networking Conference, pp. 2473-2478, Mar. 2007.

[10] W. F. Hamilton, and J. H. Rompf. "Movements of the base of the ventricle and the relative constancy of the cardiac volume," American Journal of Physiology--Legacy Content, vol. 102, no. 3, pp. 559-565, Nov. 1932.

[11] H. Suga and K. Sagawa, "Instantaneous Pressure-Volume Relationships and Their Ratio in the Excised, Supported Canine Left Ventricle," Circulation Research, vol. 35, no. 1, pp. 117-126, Jan. 1974.

[12] K. Y. Yazdandoost and R. Kohno, "UWB antenna for wireless body area network," 2006 Asia-Pacific Microwave Conference, pp. 1647-1652, Dec. 2006.

[13] K. Y. Yazdandoost and R. Kohno, "Wireless Communications for Body Implanted Medical Device," Asia-Pacific Microwave Conference, pp. 14, Dec. 2007.

[14] K. Y. Yazdandoost and R. Kohno, "Body Implanted Medical Device Communications," IEICE Transactions on Communications, vol. 92, no. 2, pp. 410-417, Feb. 2009.

[15] W.-T. Chen; H.-R. Chuang, "Numerical computation of human interaction with arbitrarily oriented superquadric loop antennas in personal communications," IEEE Trans. on Antenna and Propagation, vol.46, no. 6, pp. 821-828, June 1998.

[16] K. Y. Yazdandoost and R. Kohno, "Ultra Wideband Lloop antenna," IEEE International Conference on Ultra-Wideband, pp. 201-205, Sep. 2005.

[17] L. Chirwa, P. Hammond, S. Roy, and D. Cumming, "Electromagnetic radiation from ingested sources in the human intestine between $150 \mathrm{MHz}$ and $1.2 \mathrm{GHz}$," IEEE Transactions on Biomedical Engineering, vol. 50, no. 4, pp. 484-492, Apr. 2003.

[18] N. Cho, J. Yoo, S.-J. Song, J. Lee, S. Jeon, and H.-J. Yoo, "The Human Body Characteristics as a Signal Transmission Medium for Intrabody Communication," IEEE Transactions on Microwave Theory and Techniques, vol. 55, no. 5, pp. 1080-1086, May 2007.

[19] Y. El-Saboni, G. A. Conway, and W. G. Scanlon, "Effect of tissue boundaries on the intra-body communication channel at $2.38 \mathrm{GHz}$," International Workshop on Antenna Technology: Small Antennas, Innovative Structures, and Applications (iWAT), pp. 285-288, Mar. 2017.

[20] J. C. Bamber, "Attenuation and Absorption," Physical Principles of Medical Ultrasonics, vol 2., pp. 93-166, Mar. 2004.

[21] C. Johnson and A. Guy, "Nonionizing electromagnetic wave effects in biological materials and systems," Proceedings of the IEEE, vol. 60, no. 6, pp. 692-718, Jun. 1972.

[22] L.F. Chen, C.K. Ong, C.P. Neo, V.V. Varadan, V.K. Varadan, "Microwave electronics: measurement and materials characterization," John Wiley \& Sons; Nov 2004.

[23] A. Zangwill, "Physics at surfaces," Cambridge university press, Mar. 1988.

[24] Microwave Studio, "CST-Computer Simulation Technology." Bad Nuheimer Str, vol. 19,pp. 64289, 2008.

[25] I. Dove, "Analysis of radio propagation inside the human body for in-body Localization purposes," 2014.

[26] K. S. Cole and R. H. Cole, "Dispersion and Absorption in Dielectrics I. Alternating Current Characteristics," The Journal of Chemical Physics, vol. 9, no. 4, pp. 341-351, Apr. 1941. 
[27] K. Sasaki, K. Wake, and S. Watanabe, "Development of best fit Cole-Cole parameters for measurement data from biological tissues and organs between $1 \mathrm{MHz}$ and $20 \mathrm{GHz}$," Radio Science, vol. 49, no. 7, pp. 459-472, Jul. 2014.

[28] H. J. Visser, "Taking ECG-signals from a human voxel model," Loughborough Antennas \& Propagation Conference (LAPC), pp. 1-5, Nov. 2015.

[29] K.R. Foster and H.P. Schwan, "Dielectric properties of tissues," Handbook of biological effects of electromagnetic fields, pp.25102, 1995.

[30] M. Ackerman, "The Visible Human Project," Proceedings of the IEEE, vol. 86, no. 3, pp. 504-511, Mar. 1998.

[31] V. Spitzer, M. Ackerman, A. Scherzinger and D. Whitlock, "The Visible Human Male: A Technical Report", Journal of the American Medical Informatics Association, vol. 3, no. 2, pp. 118-130, Mar. 1996.

[32] A. M. Maceira, S. K. Prasad, M. Khan, and D. J. Pennell, "Reference right ventricular systolic and diastolic function normalized to age, gender and body surface area from steady-state free precession cardiovascular magnetic resonance," European Heart Journal, vol. 27, no. 23, pp. 28792888, Nov. 2006.

[33] V. Spitzer, M. Ackerman, A. Scherzinger and D. Whitlock, "The Visible Human Male: A Technical Report", Journal of the American Medical Informatics Association, vol. 3, no. 2, pp. 118-130, Mar. 1996.

[34] A. Khaleghi and I. Balasingham, "Power coupling for conceptual antennas in medical implant applications," 11th European Conference on Antennas and Propagation (EUCAP), pp. 1864-1867, Mar. 2017.

[35] A. Khaleghi and I. Balasingham, "Wireless communication link for capsule endoscope at $600 \mathrm{MHz}, " 2015$ 37th Annual International Conference of the IEEE Engineering in Medicine and Biology Society (EMBC), pp. 4081-4084, Aug. 2015. 